\title{
Efficient Design Optimization of Complex Electromagnetic Systems using Parametric Macromodeling Techniques
}

\author{
Francesco Ferranti*, Giulio Antonini ${ }^{\dagger}$, Tom Dhaene*, Luc Knockaert*, Antonio Ciccomancini Scogna ${ }^{\ddagger}$ \\ *Department of Information Technology, Ghent University-IBBT, Gaston Crommenlaan 8 Bus 201, B-9050, Ghent, Belgium \\ Email: francesco.ferranti@intec.ugent.be; tom.dhaene@intec.ugent.be; luc.knockaert@intec.ugent.be \\ ${ }^{\dagger}$ Dipartimento di Ingegneria Elettrica e dell’Informazione, Università degli Studi dell’Aquila, 67100, L'Aquila, Italy \\ Email: giulio.antonini@univaq.it \\ ${ }^{\ddagger}$ CST of America, 492 Old Connecticut Path, Suite 505, Framingham, MA, 01701, USA \\ E-mail: Antonio.Ciccomancini@cst.com.
}

\begin{abstract}
We propose a new parametric macromodeling technique for complex electromagnetic systems described by scattering parameters, which are parameterized by multiple design variables such as layout or substrate feature. The proposed technique is based on an efficient and reliable combination of rational identification, a procedure to find scaling and frequency shifting system coefficients, and positive interpolation schemes. Parametric macromodels can be used for efficient and accurate design space exploration and optimization. A design optimization example for a complex electromagnetic system is used to validate the proposed parametric macromodeling technique in a practical design process flow.
\end{abstract}

\section{INTRODUCTION}

During a typical design process of electromagnetic (EM) systems, design space exploration, design optimization and sensitivity analysis are usually performed and require multiple frequency-domain simulations for different design parameter values (e.g. layout features). Parametric macromodels are suitable to efficiently and accurately perform these design activities, while using multiple EM simulations may often be high computationally expensive due to the high computational cost per simulation. Parametric macromodels are multivariate models that describe the complex behavior of EM systems, which is typically characterized by the frequency (or time) and several design parameters, such as layout or substrate features.

Over the last years, different parametric macromodeling techniques have been developed. In [1], [2], both poles and residues are parameterized and it results in accurately modeling dynamic multivariate data. Overall stability and passivity of parametric macromodels are not guaranteed. Only residues are parameterized in [3], [4] and therefore the modeling capability is reduced in comparison with [1], [2], but the stability and passivity of parametric macromodels are guaranteed. Recently, new parametric macromodeling methods in [5]-[7] overcome some limitations of [1], [2] and [3], [4], since a parameterization of poles and residues is performed, while preserving stability and passivity over the entire design space. Unfortunately, these methods are sensitive to some issues related to the interpolation of state-space matrices [8] and can only deal with rational models of the same order. The matrix solution of positive-real and bounded-real lemma are used to perform a passivity preserving interpolation of state-space matrices. Its computation can be carried out using Linear Matrix Inequalities (LMI) or Riccati equation solvers. The complexity of LMI computations can grow quickly with the number of states $n$. For example, the number of operations required to solve a Riccati equation is $O\left(n^{3}\right)$, while the cost of solving an equivalent LMI is $O\left(n^{6}\right)$ [9].

This paper presents a new parametric macromodeling method for scattering $(\mathbf{S})$ representations, which indirectly parameterizes poles and residues and is able to guarantee overall stability and passivity. Initially, a set of univariate frequencydependent macromodels related to different values of the design variables, called root macromodel [3], [4], is built by means of the Vector Fitting (VF) technique [10]. Stability for each root macromodel is enforced by pole-flipping [10], while passivity is checked and enforced by means of standard techniques (see e.g. [11], [12]). Next, the computation and parameterization of scaling and frequency shifting system coefficients for each root macromodel is performed. Finally, a parametric macromodel is obtained by a combination of root macromodels and corresponding scaling and frequency shifting coefficients, using positive interpolation schemes that preserve stability and passivity over the complete design space. The proposed technique can deal with root macromodels of different orders, it is able to guarantee overall passivity and stability without solving positive-real and bounded-real lemma and is not sensitive to the well-known issues related to the interpolation of state-space matrices. The proposed method is used in the optimization process of a complex electromagnetic systems; the numerical results show that the parametric 
macromodel allows significant speed-ups with respect to EM simulations and, thus, it is well suited to be applied to a real design process flow.

\section{PARAmetric Macromodeling}

The proposed method aims at building a parametric macromodel $\mathbf{R}(s, \boldsymbol{g})$ that accurately describes a set of data samples $\left\{(s, \boldsymbol{g})_{k}, \mathbf{H}(s, \boldsymbol{g})_{k}\right\}_{k=1}^{K_{t o t}}$ and guarantees stability and passivity over the entire design space. These multivariate data samples depend on the complex frequency $s=j \omega$ and several design variables $\boldsymbol{g}=\left(g^{(m)}\right)_{m=1}^{M}$, such as layout features or substrate parameters. A parametric macromodel in the form

$$
\mathbf{R}(s, \boldsymbol{g})=\mathbf{C}(\boldsymbol{g})(s \mathbf{I}-\mathbf{A}(\boldsymbol{g}))^{-1} \mathbf{B}(\boldsymbol{g})+\mathbf{D}(\boldsymbol{g})
$$

is computed by the proposed parametric macromodeling method. Two data grids are used in the modeling process: an estimation grid and a validation grid. The estimation grid is utilized to build the root macromodels. The validation grid is used to validate the modeling capability of the parametric macromodel in a set of points of the design space previously not used for the construction of the root macromodels. The design space $\mathcal{D}(\boldsymbol{g})$ is considered as the parameter space $\mathcal{P}(s, \boldsymbol{g})$ without frequency. The parameter space $\mathcal{P}(s, \boldsymbol{g})$ contains all parameters $(s, \boldsymbol{g})$. If the parameter space is $(\mathbf{M}+1)$ dimensional, the design space is M-dimensional.

\section{A. Root Macromodels}

Starting from a set of data samples $\left\{(s, \boldsymbol{g})_{k}, \mathbf{H}(s, \boldsymbol{g})_{k}\right\}_{k=1}^{K_{t o t}}$, a set of frequency-dependent rational macromodels is built in the estimation design space grid by means of the VF technique [10]. A pole-flipping scheme is used to enforce stability [10], while passivity assessment and enforcement can be accomplished using the robust standard techniques [11], [12]. This initial procedure results in a set of stable and passive rational univariate macromodels that we call root macromodels.

\section{B. Scaling and Frequency Shifting Coefficients}

After the computation of root macromodels, the next step is gluing them together and building a multivariate representation $\mathbf{R}(s, \boldsymbol{g})$. The design space is divided into cells using hyperrectangles (regular grid) [13] or simplices (regular and scattered grid) [14], [15]. Fig. 1 shows a possible 2-D design space divided into cells, in the regular and scattered case, respectively.

Once the design space is divided into cells, a local parametric model is associated to every cell that is a subdomain of the entire design space. We indicate a cell region of the design space as $\Omega_{i}, i=1, \ldots, P$ and the corresponding vertices as $\boldsymbol{g}_{k}^{\Omega_{i}}, \quad k=1, \ldots, Q$. We note that each vertex corresponds to a root macromodel $\mathbf{R}\left(s, \boldsymbol{g}_{k}^{\Omega_{i}}\right)$. For each cell, an optimization procedure is used to find the scaling and frequency shifting system coefficients that make each vertex an accurate approximant of the other cell vertices. For each vertex $\mathbf{R}\left(s, \boldsymbol{g}_{k}^{\Omega_{i}}\right)$, a set of scaling $\alpha_{1, k}\left(\boldsymbol{g}_{j}^{\Omega_{i}}\right), j=1, \ldots, Q$ and
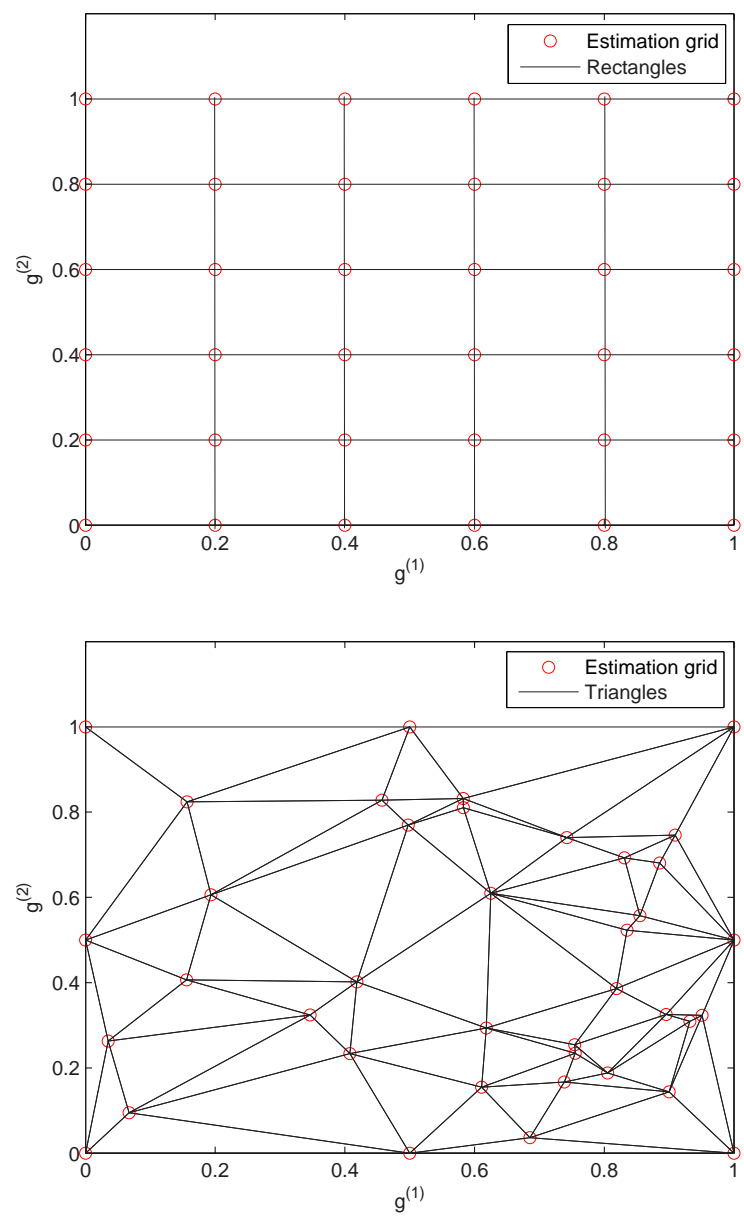

Fig. 1. Design space divided into cells: regular (top) and scattered (bottom).

frequency shifting $\alpha_{2, k}\left(\boldsymbol{g}_{j}^{\Omega_{i}}\right), j=1, \ldots, Q$ real coefficients are found, such that

$$
\begin{gathered}
\alpha_{1, k}\left(\boldsymbol{g}_{j}^{\Omega_{i}}\right) \mathbf{R}\left(s \alpha_{2, k}\left(\boldsymbol{g}_{j}^{\Omega_{i}}\right), \boldsymbol{g}_{k}^{\Omega_{i}}\right) \simeq \mathbf{R}\left(s, \boldsymbol{g}_{j}^{\Omega_{i}}\right), j \neq k \\
\alpha_{1, k}\left(\boldsymbol{g}_{j}^{\Omega_{i}}\right)=\alpha_{2, k}\left(\boldsymbol{g}_{j}^{\Omega_{i}}\right)=1, j=k
\end{gathered}
$$

If the response of the system under modeling needs to be computed in a specific design space point $\widehat{\boldsymbol{g}}$, a subdomain that contains $\widehat{\boldsymbol{g}}$ is to be found. For each vertex root macromodel of the found subdomain, the corresponding sets of scaling and frequency shifting coefficients $\alpha_{1, k}\left(\boldsymbol{g}_{j}^{\Omega_{i}}\right), \alpha_{2, k}\left(\boldsymbol{g}_{j}^{\Omega_{i}}\right)$ are interpolated in $\widehat{\boldsymbol{g}}$ and a rational model $\widehat{\alpha}_{1, k} \mathbf{R}\left(s \widehat{\alpha}_{2, k}, \boldsymbol{g}_{k}^{\Omega_{i}}\right)$ is built, where $\widehat{\alpha}_{1, k}=\alpha_{1, k}(\widehat{\boldsymbol{g}})$ and $\widehat{\alpha}_{2, k}=\alpha_{2, k}(\widehat{\boldsymbol{g}})$. Finally, the set of modified root macromodels $\widehat{\alpha}_{1, k} \mathbf{R}\left(s \widehat{\alpha}_{2, k}, \boldsymbol{g}_{k}^{\Omega_{i}}\right), k=1, \ldots, Q$, is interpolated at an input/output level as described in [3], [4]. We note that if a generic root macromodel $\mathbf{R}\left(s, \boldsymbol{g}_{k}^{\Omega_{i}}\right)$ has the state-space representation $\{\mathbf{A}, \mathbf{B}, \mathbf{C}, \mathbf{D}\}$, then a corresponding scaled and frequency shifted version $\widehat{\alpha}_{1, k} \mathbf{R}\left(s \widehat{\alpha}_{2, k}, \boldsymbol{g}_{k}^{\Omega_{i}}\right)$ has the state-space representation $\{\widetilde{\mathbf{A}}, \widetilde{\mathbf{B}}, \widetilde{\mathbf{C}}, \widetilde{\mathbf{D}}\}$ with 


$$
\begin{aligned}
\widetilde{\mathbf{A}} & =\left(\widehat{\alpha}_{2, k}\right)^{-1} \mathbf{A} \\
\widetilde{\mathbf{B}} & =\mathbf{B} \\
\widetilde{\mathbf{C}} & =\widehat{\alpha}_{1, k}\left(\widehat{\alpha}_{2, k}\right)^{-1} \mathbf{C} \\
\widetilde{\mathbf{D}} & =\widehat{\alpha}_{1, k} \mathbf{D}
\end{aligned}
$$

\section{Multivariate Interpolation of Scaling, Frequency Shifting Coefficients and Root Macromodels}

Passivity refers to the property of systems that cannot generate more energy than they absorb through their electrical ports. When the system is terminated on any arbitrary passive loads, none of them will cause the system to become unstable [16]. Passivity is crucial when the macromodel is utilized in a circuit simulator (e.g. SPICE [17]) for transient analysis. The passivity of scattering input-output representations is also called nonexpansivity [18] A linear network described by scattering matrix $\mathbf{S}(s)$ is passive if [19]:

1) $\mathbf{S}\left(s^{*}\right)=\mathbf{S}^{*}(s)$ for all $s$, where "*" is the complex conjugate operator.

2) $\mathbf{S}(s)$ is analytic in $\Re e(s)>0$.

3) $\mathbf{I}-\mathbf{S}^{t}\left(s^{*}\right) \mathbf{S}(s) \geq 0 ; \forall s: \Re e(s)>0$.

Condition 3) for nonexpansivity is equivalent to the condition $\|\mathbf{R}(s)\|_{\infty} \leq 1$ ( $\mathbf{H}_{\infty}$ norm) [18], i.e., the largest singular value of $\mathbf{R}(s)$ does not exceed one in the right-half $s$-plane. The interpolated scaling and frequency shifting real coefficients $\alpha_{1, k}(\boldsymbol{g}), \alpha_{2, k}(\boldsymbol{g})$ have to satisfy the conditions

$$
\begin{aligned}
0 \leq \alpha_{1, k}(\boldsymbol{g}) & \leq 1 \\
\alpha_{2, k}(\boldsymbol{g}) & \geq 0
\end{aligned}
$$

to guarantee the passivity of each root macromodel $\alpha_{1, k}(\boldsymbol{g}) \mathbf{R}\left(s \alpha_{2, k}(\boldsymbol{g}), \boldsymbol{g}_{k}^{\Omega_{i}}\right)$.

Multivariate interpolation based on a class of positive interpolation operators [20], [21] is used to parameterize $\alpha_{1, k}(\boldsymbol{g}), \alpha_{2, k}(\boldsymbol{g})$. It is able to guarantee the passivity of each scaled and frequency shifted root macromodel over the entire design space by guaranteeing the properties (5a)(5b). The same positive multivariate interpolation schemes are used to interpolate the set of modified root macromodels $\widehat{\alpha}_{1, k} \mathbf{R}\left(s \widehat{\alpha}_{2, k}, \boldsymbol{g}_{k}^{\Omega_{i}}\right), \quad k=1, \ldots, Q$, at an input/output level, which results in a parametric macromodel, stable and passive over the entire design space.

In the bivariate case $(s, g)$, each interpolated function $\mathbf{T}(g)$ can be written as

$$
\mathbf{T}(g)=\sum_{k=1}^{K_{1}} \mathbf{T}_{g_{k}} \ell_{k}(g)
$$

where $K_{1}$ represents the number of root macromodels and each interpolation kernel $\ell_{k}(g)$ is a scalar function satisfying the following constraints

$$
\begin{gathered}
0 \leq \ell_{k}(g) \leq 1, \\
\ell_{k}\left(g_{i}\right)=\delta_{k, i}, \\
\sum_{k=1}^{K_{1}} \ell_{k}(g)=1 .
\end{gathered}
$$

A possible choice is to select $\ell_{k}(g)$ as in piecewise linear interpolation

$$
\begin{gathered}
\frac{g-g_{k-1}}{g_{k}-g_{k-1}}, g \in\left[g_{k-1}, g_{k}\right], k=2, \ldots, K_{1}, \\
\frac{g_{k+1}-g}{g_{k+1}-g_{k}}, g \in\left[g_{k}, g_{k+1}\right], k=1, \ldots, K_{1}-1, \\
0 \quad, \text { otherwise }
\end{gathered}
$$

In the general multivariate case, multivariate interpolation methods that belong to the general class of positive interpolation operators can be used, e.g., the piecewise multilinear and multivariate simplicial methods [13]. We note that the interpolation kernel functions of these methods only depend on the design space grid points and their computation does not require the solution of a linear system to impose an interpolation constraint. In the case of piecewise multilinear interpolation, each interpolated function $\mathbf{T}\left(g^{(1)}, \ldots, g^{(M)}\right)$ can be written as

$$
\begin{aligned}
& \mathbf{T}\left(g^{(1)}, \ldots, g^{(M)}\right)= \\
& =\sum_{k_{1}=1}^{K_{1}} \cdots \sum_{k_{M}=1}^{K_{M}} \mathbf{T}_{\left(g_{k_{1}}^{(1)}, \ldots, g_{k_{M}}^{(M)}\right)} \ell_{k_{1}}\left(g^{(1)}\right) \cdots \ell_{k_{M}}\left(g^{(M)}\right)
\end{aligned}
$$

where each $\ell_{k_{i}}\left(g^{(i)}\right), i=1, \ldots, M$ satisfies constraints (7)(9) and is selected as in piecewise linear interpolation. These positive interpolation schemes have been already used in [3], [4], where a parametric macromodel is built by interpolating a set of root macromodels treated as input-output systems, while preserving overall stability and passivity. In the proposed new parametric macromodeling technique, a powerful novelty is the use of some interpolated scaling and frequency shifting system coefficients. It allows to parameterize poles and residues indirectly, hence the modeling capability of the proposed algorithm is increased with respect to [3], [4], where the interpolation process were only applied to the root macromodels, and therefore only residues were parameterized.

\section{Passivity Preserving Interpolation}

In this section, we prove that a passive system $\mathbf{R}(s)$ remains passive if a scaling coefficient $\alpha_{1}$ and a frequency shifting coefficient $\alpha_{2}$, which satisfy the properties (5a)-(5b), are applied to it. The scaling coefficient $\alpha_{1}$ is a multiplicative factor at the input/output level of the system, while the frequency shifting coefficient $\alpha_{2}$ is a compression or expansion term for the Laplace variable $s$. It is easy to prove that if $\alpha_{2}$ satisfies (5b), passivity is preserved, and that if $\alpha_{1}$ satisfies (5a), the first two conditions for passivity are preserved. Concerning $\alpha_{1}$ and the third passivity condition 


$$
\left\|\alpha_{1} \mathbf{R}\left(\alpha_{2} s\right)\right\|_{\infty}=\alpha_{1}\left\|\mathbf{R}\left(\alpha_{2} s\right)\right\|_{\infty} \leq \alpha_{1} \leq 1
$$

Therefore, if $\alpha_{1}$ satisfies (5a), passivity is preserved. The proof of the passivity preserving interpolation of root macromodels can be found in [3], [4].

\section{OptimizATION}

Once the parametric macromodel is built, it can be used in an optimization process. The optimization of EM systems involves formulation of cost functions related to the frequency behavior. A general cost function can be represented as

$$
\mathbf{F}_{i}(\boldsymbol{g})=\mathbf{R}_{L i}-\mathbf{R}\left(s_{i}, \boldsymbol{g}\right)
$$

or

$$
\mathbf{F}_{i}(\boldsymbol{g})=\mathbf{R}\left(s_{i}, \boldsymbol{g}\right)-\mathbf{R}_{U i}
$$

with $i=1, \ldots, N_{s}$, where $N_{s}$ denotes the number of frequency samples, $\mathbf{R}_{L i}$ and $\mathbf{R}_{U i}$ represent lower and upper frequency response thresholds, respectively. A negative error value indicates that the corresponding specification is satisfied, while a positive error value denotes that the specification is violated. The minimization of the cost functions (15)-(16) can be performed by several optimization algorithms. In this paper, we use a minimax optimization algorithm [22] that provides the optimum set of design parameter values $\widetilde{\boldsymbol{g}}$

$$
\widetilde{\boldsymbol{g}}=\underset{\boldsymbol{g}}{\operatorname{argmin}}\left\{\max _{i}\left[\mathbf{F}_{i}(\boldsymbol{g})\right]\right\}
$$

\section{NUMERICAL RESULTS}

This section presents a design optimization example to validate the proposed method in a real design process flow. Let us define the absolute error

$$
\begin{aligned}
& \operatorname{Err}(\boldsymbol{g})=\max \left(\mid\left(R_{i, j}\left(s_{k}, \boldsymbol{g}\right)-H_{i, j}\left(s_{k}, \boldsymbol{g}\right) \mid\right)\right. \\
& i=1, \ldots, P_{\text {in }}, j=1, \ldots, P_{\text {out }}, k=1, \ldots, K_{s}
\end{aligned}
$$

where $P_{\text {in }}$ and $P_{\text {out }}$ are the number of inputs and outputs of the system, respectively, and $K_{s}$ is equal to the number of frequency samples. The worst case absolute error over the validation grid is chosen to assess the accuracy and the quality of parametric macromodels

$$
\begin{gathered}
\boldsymbol{g}_{\text {max }}=\underset{\boldsymbol{g}}{\operatorname{argmax}} \operatorname{Err}(\boldsymbol{g}), \boldsymbol{g} \in \text { validation grid } \\
\qquad \operatorname{Er}_{\max }=\operatorname{Err}\left(\boldsymbol{g}_{\max }\right)
\end{gathered}
$$

The number of poles for each root macromodel is selected adaptively in VF by a bottom-up approach, in such a way that the corresponding maximum absolute error is smaller than $-60 \mathrm{~dB}$.

\section{3-D example: SMA structure}

Two SMA launch connecting a single ended stripline in a fourteen layer PCB are modeled and optimized in this example. The trace is 549 mils long, 5 mils wide and 0.6 mils tick and it is routed on the fourth layer, in order to also consider the effect of the via stub related to the inner conductor of each SMA. The width $(W)$ of the trace and the radius $(R)$ of the seven grounding vias surrounding the SMA are used as parameters to optimize the performance of the launch. In order to excite a pure TEM mode, and therefore generate a meaningful scattering matrix, a waveguide port is used on the top of the SMA to excite the structure. The dielectric material used for the PCB stack-up is Nelco with permittivity of $\epsilon_{r}=3.5$ and loss tangent $\operatorname{tg}(\delta)=0.009$, whereas the metal is copper. Fig. 2 shows the structure under modeling. A trivariate reduced order model is built as a function of frequency, the with of the trace and the radius of the grounding vias. Table I shows their corresponding ranges.

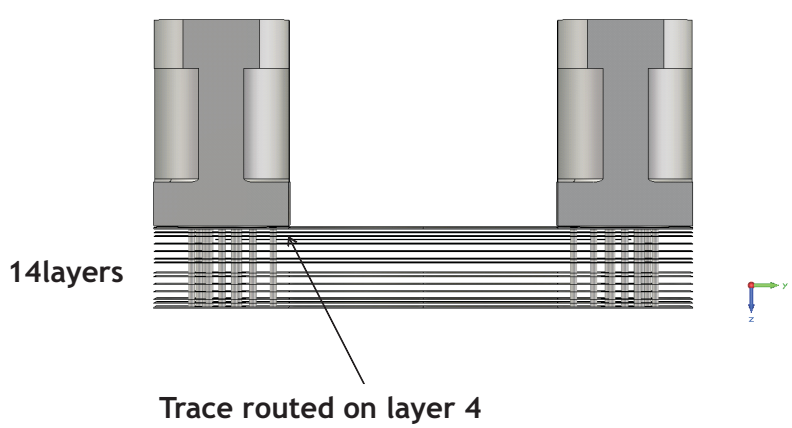

Fig. 2. SMA structure.

TABLE I

PARAMETERS OF SMA STRUCTURE.

\begin{tabular}{|l|c|c|}
\hline Parameter & Min & Max \\
\hline Frequency (freq) & $0 \mathrm{~Hz}$ & $30 \mathrm{GHz}$ \\
Trace width (W) & $30.5 \mathrm{mils}$ & $34.5 \mathrm{mils}$ \\
Vias Radius (R) & $3 \mathrm{mils}$ & $5 \mathrm{mils}$ \\
\hline
\end{tabular}

The scattering parameters have been computed by means of the commercial software [23] over a validation grid of $135 \times 13 \times 13$ samples, for frequency, $W$ and $R$, respectively. We have built root macromodels for 6 values of $W$ and 6 values of $R$ by means of VF, each with an order chosen by the error-based bottom-up approach described in Section IV. Finally, a trivariate macromodel is obtained as explained in Section II, using multilinear interpolation for the scaling, shifting coefficients and root macromodels. Figs. 3-4 show the magnitude of the parametric macromodels of $\mathbf{S}_{11}(s, W, R)$ for the vias radius $R=32.17$ mils and $\mathbf{S}_{21}(s, W, R)$ for the trace width value $W=3.83$ mils. Fig. 5 compares $\mathbf{S}_{11}(s, W, R)$ and its macromodel for the values $R=\{30.83,12.17,33.5\}$ mils, $W=3.83$ mils that have not been used for the generation of the root macromodels. The worst case absolute 
error defined in (20) is equal to $-35 \mathrm{~dB}$ and it occurs for $g_{\max }=\{W, R\}=\{3.83$ mils, 32.17 mils $\}$.

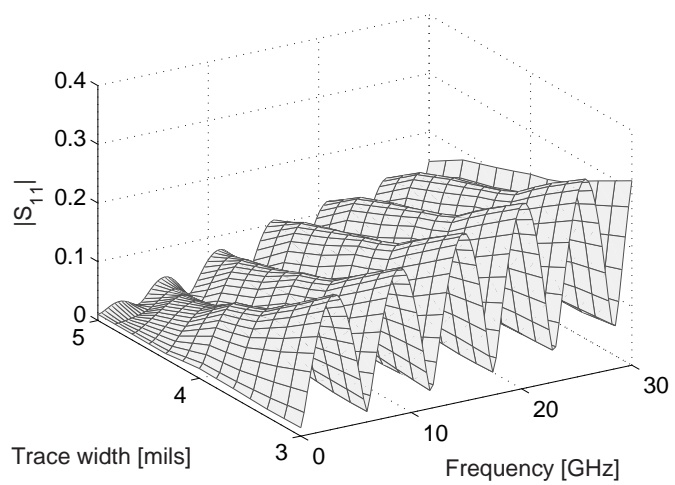

Fig. 3. Magnitude of the trivariate macromodel of $\mathbf{S}_{11}(s, W, R)$ for $R=$ 32.17 mils

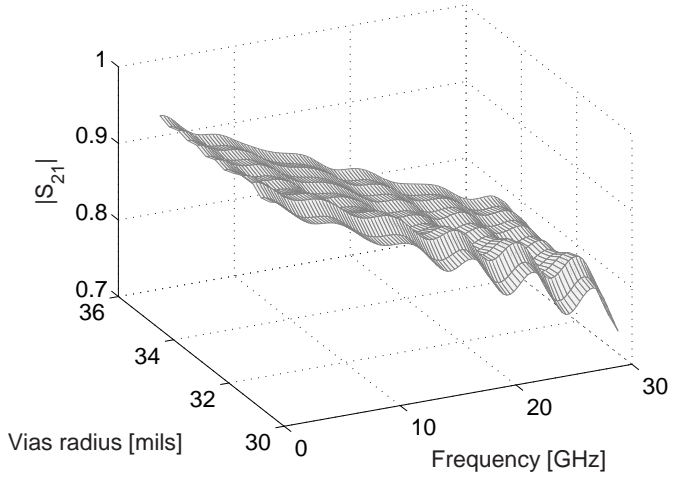

Fig. 4. Magnitude of the trivariate macromodel of $\mathbf{S}_{21}(s, W, R)$ for $W=$ 3.83 mils.

The parametric macromodel is able to accurately describe the behavior of the system, while stability and passivity are guaranteed over the entire design space. Once the parametric macromodel is built, it is used in an optimization step. The objective function is ensuring that the magnitude of $\mathbf{S}_{11}$ is below $-20 \mathrm{~dB}$ in the bandwidth of interest. A minimax algorithm is used for the optimization. The starting values $g_{\max }=\{W, R\}=\{3.83$ mils, 32.17 mils $\}$ are used for the optimization and the optimal values are found to be $\widetilde{\boldsymbol{g}}=\{W, R\}=\{4.94$ mils, 34.5 mils $\}$. Table II shows the computational time needed to perform the optimization by means of the commercial software [23] and the parametric macromodel. We note that the computation and validation of the parametric macromodel requires an initial set of simulations by means of [23] and therefore an initial computational effort, but once the parametric macromodel is created and validated, it becomes an accurate and efficient surrogate of the original system and can be used for every optimization that involves the modeled system in the design space defined during the construction of the parametric macromodel.
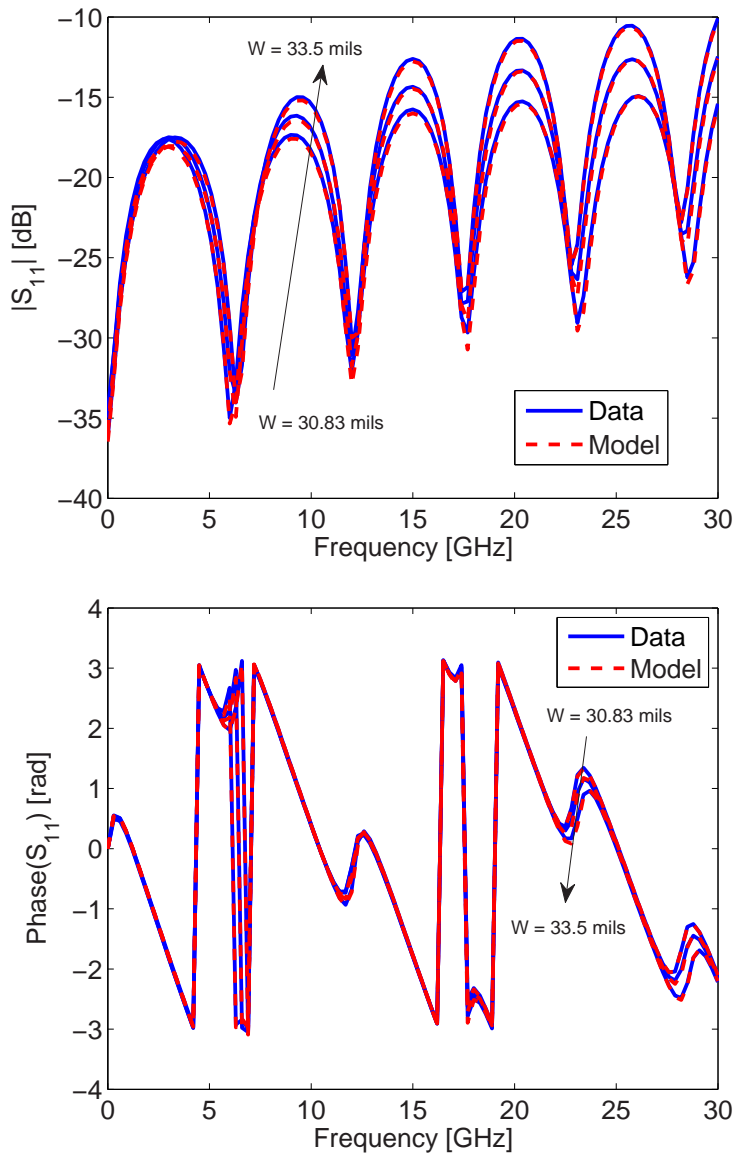

Fig. 5. Magnitude and the phase of the trivariate macromodel of $\mathbf{S}_{11}(s, W, R)(R=\{30.83,12.17,33.5\}$ mils, $W=3.83$ mils $)$.

TABLE II

CPU TIME FOR THE OPTIMIZATION.

\begin{tabular}{|l|c|}
\hline Method & CPU time \\
\hline CST Studio & $33 \mathrm{~h}$ \\
Parametric macromodel & $9.38 \mathrm{~s}$ \\
\hline
\end{tabular}

As clearly seen, the obtained speed up confirms the applicability of the proposed parametric macromodeling techniques to accurate and efficient design optimization. Fig. 6 shows the initial and optimized $\mathbf{S}_{11}$ response.

\section{CONCLUSions}

We have presented a new parametric macromodeling method for scattering representations. Poles and residues are parameterized indirectly, while overall stability and passivity are guaranteed. The proposed method is based on an efficient and reliable combination of rational identification, a procedure to find scaling and frequency shifting system coefficients, and positive interpolation schemes. The proposed method is used in the optimization process of a complex electromagnetic system, which shows the speed up obtained using the parametric macromodel instead of EM simulations and its applicability 


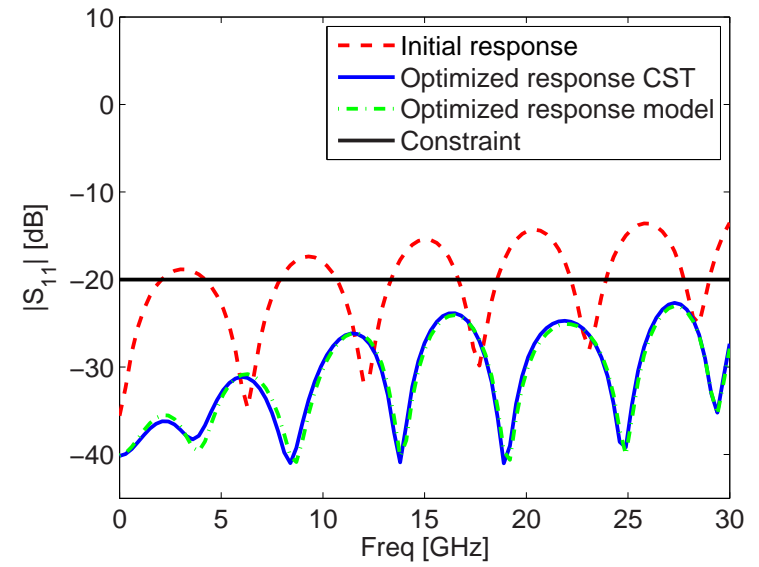

Fig. 6. Optimization of $\mathbf{S}_{11}$.

to a real design process flow.

\section{ACKNOWLEDGMENT}

This work was supported by the Research Foundation Flanders (FWO).

\section{REFERENCES}

[1] D. Deschrijver, T. Dhaene and D. De Zutter, "Robust parametric macromodeling using multivariate orthonormal vector fitting," IEEE Trans. Microw. Theory Tech., vol. 56, no. 7, pp. 1661-1667, Jul. 2008.

[2] P. Triverio, S. Grivet-Talocia, M. S. Nakhla, "An improved fitting algorithm for parametric macromodeling from tabulated data," in Proc. in Workshop Signal Propagation on Interconnects, Avignon, France, May 2008, pp. 1-4.

[3] F. Ferranti, L. Knockaert, and T. Dhaene, "Guaranteed passive parameterized admittance-based macromodeling," IEEE Trans. Adv. Packag., vol. 33, no. 3, pp. 623-629, Aug. 2010.

[4] _ - "Parameterized S-parameter based macromodeling with guaranteed passivity," IEEE Microw. Wireless Compon. Lett., vol. 19, no. 10, pp. 608-610, Oct. 2009.

[5] F. Ferranti, L. Knockaert, T. Dhaene, G. Antonini, and D. De Zutter, "Parametric macromodeling for tabulated data based on internal passivity," IEEE Microw. Wireless Compon. Lett., vol. 20, no. 10, pp. 533 535, Oct. 2010.

[6] F. Ferranti, L. Knockaert, T. Dhaene, and G. Antonini, "Passivitypreserving parametric macromodeling for highly dynamic tabulated data based on Lur'e equations," IEEE Trans. Microw. Theory Tech., vol. 58, no. 12 , pp. $3688-3696$, Dec. 2010.

[7] P. Triverio, M. Nakhla, and S. Grivet-Talocia, "Extraction of parametric circuit models from scattering parameters of passive RF components," in European Microwave Conference (EuMC), Sept. 2010, pp. 1635 -1638.

[8] J. De Caigny, J. F. Camino, and J. Swevers, "Interpolating model identification for SISO linear parameter-varying systems," Mechanical Systems and Signal Processing, vol. 23, no. 8, pp. 2395 - 2417, 2009.

[9] G. Balas, R. Chiang, A. Packard, and M. Safonov, "Robust Control Toolbox User's Guide, Version 3," The Mathworks, Inc., 2005.

[10] B. Gustavsen and A. Semlyen, "Rational approximation of frequency domain responses by vector fitting," IEEE Trans. Power Delivery, vol. 14, no. 3, pp. 1052-1061, Jul. 1999.

[11] D. Saraswat, R. Achar and M. Nakhla, "On passivity enforcement for macromodels of S-parameter based tabulated subnetworks," in Proc. of the IEEE Int. Symp. on Circuits and Systems, vol. 4, May 2005, pp. 3777-3780.

[12] T. Dhaene, D. Deschrijver, and N. Stevens, "Efficient algorithm for passivity enforcement of S-parameter based macromodels," IEEE Trans. Microw. Theory Tech., vol. 57, no. 2, pp. 415-420, Feb. 2009.
[13] W. A. Weiser and S. E. Zarantonello, "A note on piecewise linear and multilinear table interpolation in many dimensions," Mathematics of Computation, vol. 50, no. 181, pp. 253-264, Jan. 1988.

[14] H. W. Kuhn, "Some combinatorial lemmas in topology," IBM Journal of Research and Development, vol. 4, no. 5, pp. 518-524, 1960.

[15] D. F. Watson, "Computing the n-dimensional Delaunay tessellation with application to Voronoi polytopes," The Computer Journal, vol. 24, no. 2, pp. 167-172, Febr. 1981.

[16] E. A. Guillemin, Synthesis of Passive Networks. John Wiley and Sons, New York, 1957.

[17] L. W. Nagel, "SPICE: A computer program to simulate semiconductor circuits," University of California, Berkeley, Electr. Res. Lab. Report ERL M520, May 1975.

[18] S. Boyd, L. El Ghaoui, E. Feron and V. Balakrishnan, Linear Matrix Inequalities in System and Control Theory. Philadelphia, PA: SIAM, 1994, vol. 15

[19] B. D. Anderson, S. Vongpanitlerd, Network Analysis and Synthesis. Englewood Cliffs, NJ: Prentice-Hall, 1973.

[20] G. Allasia, "A class of interpolating positive linear operators: Theoretical and computational aspects," in Recent Developments in Approximation Theory, Wavelets and Applications, S. P. Singh, Ed. Dordrecht: Kluwer, 1995, pp. 1-36.

[21] G. Allasia, "Simultaneous interpolation and approximation by a class of multivariate positive operators," Numerical Algorithms, vol. 34, no. 2, pp. 147-158, Dec. 2003.

[22] D.-Z. Du and P. M. Pardalos, Minimax and Applications. Kluwer Academic Publishers, 1995.

[23] CST MICROWAVESTUDIO, www.cst.com. 